\title{
The role of social signals in segmenting observed actions in eighteen-month-old children
}

\author{
Christian Kliesch ${ }^{1,2,3}$, Eugenio Parise ${ }^{3}$, Vincent Reid ${ }^{3,4}$, \& Stefanie \\ Hoehl $^{2,5}$ \\ ${ }^{1}$ Department of Psychology, Johannes-Gutenberg-University Mainz \\ 2 Max Planck Institute for Human Cognitive and Brain Sciences \\ ${ }^{3}$ Department of Psychology, Lancaster University \\ ${ }^{4}$ School of Psychology, University of Waikato \\ ${ }^{5}$ Department of Developmental and Educational Psychology, University of Vienna
}

\begin{abstract}
Learning about actions requires children to identify the boundaries of an action and its units. Whereas some action units are easily identified, parents can support children's action learning by adjusting the presentation and using social signals. However, currently little is understood regarding how children use these signals to learn actions.

In the current study we investigate the possibility that communicative signals are a particularly suitable cue for segmenting events. We investigated this hypothesis by presenting 18 -month-old children $(\mathrm{N}=60)$ with short action sequences consisting of toy animals either hopping or sliding across a board into a house, but interrupting this two-step sequence either (a) using an ostensive signals as a segmentation cue, (b) using a non-ostensive segmentation cue, and (c) without additional segmentation information between the actions. Marking the boundary using communicative signals increased children's imitation of the less salient sliding action. Imitation of the hopping action remained unaffected. Crucially, marking the boundary of both actions using a non-communicative control condition did not increase imitation of either action. Communicative signals might be particularly suitable in segmenting non-salient actions that would otherwise be perceived as part of another action or as non-intentional. These results provide evidence of the importance of ostensive signals at event boundaries in scaffolding children's learning.
\end{abstract}

Keywords: Action Segmentation, Communicative Signals, Ostensive Communication, Action Imitation

Word count: 6010

Research highlights: 
- We investigate the role of direct gaze and infant directed speech in segmenting action sequences in 18-month-old children.

- Children are more likely to imitate parts of an action sequence if the boundaries of its units are marked by a short burst of gaze and infant-directed-speech, compared to a non-ostensive signal of the agent pausing and saying 'hmm', or no boundary marking.

- The increase in imitation is only seen for the non-salient action type; the salient action type shows high levels of imitation irrespective of the boundary marking condition.

- Ostensive signals act as communicative signals of temporal reference, delineating the different units of an action sequence for toddlers.

\section{Introduction}

Children are avid social learners. From the age of 9 months (Biro, 2013; Gredebäck, Stasiewicz, Falck-Ytter, Rosander, \& Hofsten, 2009; Reid et al., 2009) they anticipate the goals of other social agents and start imitating other people's actions during their second postnatal year (Jones, 2007, 2009). However, learning to predict others' actions and being able to copy others' actions comes with its own unique challenges. Just as it is important to discriminate an object from its surroundings, it is important to determine which action units are relevant to the action as a whole. Caregivers support children's learning of novel information using a wide range of behavioural adaptations and social signals. So far, parents' modifications of child directed actions have been studied without direct reference to how these adaptations may contribute to the segmentation of action sequences. There are a number of recent findings, however, that suggest that communicative signals may also contribute to the segmentation of event sequences, and may be particularly important for children's action learning. In the present study, we tested whether 18-month-old children can use a brief exposure to a communicative signal to segment an action sequence and subsequently adjust which parts of the action sequence they imitate.

Actions are intentional, goal-directed movements. These features distinguish them from incidental movements and events. Like events, most actions can be described on different levels. They are hierarchically organised, with simpler action units nested in higher-order action plans (Elman, 1990; Zacks \& Tversky, 2001). The action of making a cup of coffee, for example, may be described by said overarching goal ('Making coffee') or by the units that comprise the action, e.g. grinding the coffee, boiling the water, filling the coffee press with coffee, and subsequently water and pressing the coffee press down. These actions comprise the action sequence of the action 'making coffee', which in turn may be part of the

This work was partially supported by the International Centre for Language and Communicative Development $(\mathrm{LuCiD})$ at Lancaster University, funded by the Economic and Social Research Council (UK) [ES/L008955/1]. The data collection and travel costs of this study was partially supported by the travel exchange programme at the International Centre for Language and Communicative Development (LuCiD) funded by the Economic and Social Research Council (UK) [ES/L008955/1]. We would like to thank Liesbeth Forsthuber at Vienna University for double coding the results. The data and a reproducible manuscript are available on [https://osf.io/ea7pj/]

Correspondence concerning this article should be addressed to Christian Kliesch, Department of Psychology, Johannes-Gutenberg-University Mainz, Binger Str. 14-16, 55122 Mainz. E-mail: ck@antipattern.net 
overarching action sequence of 'preparing breakfast'. As we can see, most actions consist of different action units with their own goals and sub goals. At the same time, they do not exist as isolated events, but are part of a wider action sequence (Zacks, Kumar, Abrams, \& Mehta, 2009). In the current paper, we will therefore refer to the highest relevant level of analysis of the description as the action, which comprises of action units. Therefore, observing actions (and potentially carrying out actions Hommel, Müsseler, Aschersleben, \& Prinz, 2001) shares many features with a general description of events, enriched by goals and intentions (Zacks et al., 2009).

According to Event Segmentation Theory (Zacks et al., 2009; Zacks \& Swallow, 2007) segmenting a stream of events plays an important role in the comprehension and anticipation of event sequences (Baldwin, Baird, Saylor, \& Clark, 2001; Zacks et al., 2009, 2007, 2001). Events can be segmented based on low-level features, such as motion cues, or prior, higherorder knowledge of the event (Zacks \& Swallow, 2007). Adults are able to segment actions into finer or coarser units when requested (Zacks, 2004; Zacks et al., 2009). Already 10- to 11-month-old infants demonstrate sensitivity to event structures, looking longer at actions paused mid-stream, compared to those where the pause coincided with an event boundary (Baldwin et al., 2001). It is likely that visual features of the action stream are important contributors in identifying potential goals. Studies by Hespos, Saylor, and Grossman (2009) and Hespos, Grossman, and Saylor (2010) provide evidence that 6-month-old infants are more likely to detect changes of events with clear outcomes, compared to changes in the transitions between events, e.g. when a self-propelled ball moves across the screen bounces down, instead of up, before arriving at its goal location. It is likely that for such simple events, infants can draw upon a wide range of perceptual information, such as the acceleration and deceleration of movements or salient outcome effects.

There is also evidence that children can use higher order knowledge to predict action sequences, which subsequently becomes available for action segmentation as well. Already from an early age, infants show an understanding of actions as goal-directed (Biro, Verschoor, Coalter, \& Leslie, 2014; Csibra, 2003; Verschoor, Spapé, Biro, \& Hommel, 2013) and distinguish between actions that are carried out in an efficient and non-efficient manner in relation to the action goal (Gergely \& Csibra, 2003; Gredebäck \& Melinder, 2011). Goaldirected actions have distinct endpoints, when the goal state is achieved. Children do not blindly imitate actions exactly the way they are shown (cf. Csibra, 2008), but selectively imitate the parts of the action they deem as relevant in terms of achieving the desired goal of the action (Király, Csibra, \& Gergely, 2013). For example, they will imitate a model turning on a lamp with their head, but only if there is no other rational explanation for it (e.g. the model's hands were occupied Gergely, Bekkering, \& Kiraly, 2002; Király et al., 2013).

Caregivers adapt and adjust how they present actions to children to make them more accessible for leaning. Infant-directed actions are often presented in an exaggerated manner (Brand, Baldwin, \& Ashburn, 2002; Koterba \& Iverson, 2009; Rutherford \& Przednowek, 2012; Schaik, Meyer, Ham, \& Hunnius, 2020; Williamson \& Brand, 2014), are highly repetitive (Brand et al., 2009) and caregivers interrupt and emphasise the boundaries of action units (Williamson \& Brand, 2014). This balance between variation and repetition may be particularly suitable for learning and retaining attention (Brand et al., 2009; Goldstein et 
al., 2010; Twomey et al., 2017; Twomey \& Westermann, 2017) and increases the capacity to learn from socially presented actions, compared to actions that children only observe incidentally.

In addition, caregivers also use other social signals to ease and support infant learning. According to Natural Pedagogy Theory (Csibra \& Gergely, 2009, 2009) children have an innate sensitivity to some social signals that inform them of the presence of a communicative interaction. Natural Pedagogy argues that such interactions consist of two separate communicative intentions - one signalling the intention to communicate, the other transmitting the content of the message (c.f. Sperber \& Wilson, 1995, 2002). However, because young infants are unlikely to already possess the meta-cognitive skills to interpret these dual intentions, Csibra (2010) suggests that infants have an early sensitivity towards direct gaze, infant-directed speech and contingent interactions that signal caregivers' communicative intention. When addressed with these ostensive signals, children expect that caregivers will provide them with generalisable information (Csibra \& Shamsudheen, 2015). This very simple code-based system allows children to rapidly learn and acquire culturally-relevant knowledge (Csibra \& Gergely, 2009, 2011). Their use is not just restricted to explicit teaching contexts, but also free play (Sage \& Baldwin, 2012).

Caregivers also use a second type of communicative signals, referential signals, to link the content of an interaction to the world around them. For example, through the use of pointing and gaze following, caregivers can restrict the number of possible referents (A. Senju et al., 2008; Woodward \& Guajardo, 2002). Children start to follow pointing and gaze during their first year of life and (Morissette, Ricard, \& Décarie, 1995; A. Senju \& Csibra, 2008) and identify the targets of pointing between 15-18 months (Morissette et al., 1995). These signals fulfill an important role of providing spatial reference and thereby restrict the number of possible referents in space (cf. Clark, 2003).

Learning about actions also requires temporal information to identify potential candidate units for predicting and imitating an action sequence. Currently, there is no research into whether a similar referential signal exists in the temporal domain. Such a marker may be particularly important in segmenting events and action units that cannot reliably be identified through observation and therefore may be particularly important for event learning in general, and action learning in particular. A temporal marker to denote the beginnings and ends of actions and their units may be just as important for learning about actions in the same way that protodeclarative pointing enables the disambiguation of different objects.

Especially when the boundaries of events and actions cannot be reliably identified through observation, caregivers' use of signals or cues that help to segment individual action units could potentially support children's learning of these actions. Indeed, previous research has found that parents are more likely to look toward and address children at action boundaries (Brand, Hollenbeck, \& Kominsky, 2013; Brand, Shallcross, Sabatos, \& Massie, 2007; Williamson \& Brand, 2014). Children are more likely to imitate an action if a model looks at them during event boundaries (Williamson \& Brand, 2014) and parents reduce the amount of direct gaze at action boundaries with increasing age (and presumably knowledge) of their child (Brand et al., 2007). 
Many of these signals used by parents at event boundaries also signal the presence of communication, such as direct gaze and infant-directed speech. Direct gaze may be a particularly suitable signal to segment events and actions. Newborn infants already prefer looking at eyes with the contrast polarity of the human pupil/sclera, but not the inverse contrast polarity (Farroni et al., 2005). Faces and gaze also play an important role during early development, as during early infancy, faces are dominant stimuli (Fausey, Jayaraman, $\&$ Smith, 2016). This sensitivity and high frequency of exposure might help children to pay attention to faces as signifiers of interactions that are directed towards them. In adults, direct gaze has encountering brief periods of direct gaze appears to interrupt working memory and delays response times in a visual search task, but only for gaze periods of 100 milliseconds, not for longer exposures to direct and provide listener feedback, thereby structuring conversations in dialogue (e.g. Hömke, Holler, \& Levinson, 2017; Bavelas, Coates, \& Johnson, 2002). Because of its privileged role and potentially disruptive properties, adults' use of direct gaze and infant-directed speech at action boundaries may help children to break up and identify individual parts of an incoming stream of actions. Therefore, children might not only use ostensive signals to infer the presence of communication, but also segment an incoming event or action sequence. In the following study we investigate whether children use a social signal that is traditionally seen as an ostensive signal to segment an action stream by directly comparing the effect of a social, a non-social and a control condition without a boundary marker in segmenting a short action sequence.

An interesting case of children's understanding of action sequences is revealed in their imitation of an action's manner compared to its outcome (Carpenter, Call, \& Tomasello, 2005; Southgate, Chevallier, \& Csibra, 2009). Twelve- and 18-month-old toddlers are more likely to imitate the manner of an action in the absence of a clear outcome. When an experimenter moved a toy animal either in a hopping or sliding movement, toddlers were more likely to imitate the manner when they saw the action performed on its own, but not when they were shown the animal go into a house (Carpenter et al., 2005). It is likely that when children observe the sliding/hopping action on its own, they interpret the manner of the movement as the goal of the action. However, when the action has another, overarching goal (such as putting the animal into the house), toddlers see the manner as merely instrumental (but not essential) in achieving the goal of putting the animal into the house. If that was indeed the case, children's imitation of the action's manner might increase if the action sequence of hopping/sliding the animal into the house is interrupted between both action units, for example by addressing the children using direct gaze and infant-directed speech. This raises the interesting question of whether certain signals, such as ostensive signals, are particularly suitable to segment such an event sequence, or whether any interruption of the action sequence is sufficient to mark both action units as separate units and subsequently increase the imitation of the action's manner.

We investigated this question in a between-subjects experiment by presenting three groups of 18-month-old toddlers with a short action sequence of moving an animal into a house in two distinct manners. Previous research found that children at this age frequently imitate the outcome of the action at the expense of the manner (Carpenter et al., 2005; Southgate et al., 2009). We predicted that children, when they encounter such an action demonstration, perceive the action sequence of hopping or sliding into the house as part 
of a single action unit and consequently focus only on the outcome. However, if social signals aid children's action unit segmentation, establishing direct gaze and addressing the child with infant-directed speech at the boundary between the hopping/sliding event and putting the animal into the house should help them interpret both action units as separate units worthy of imitation. We predicted that, if segmented in such way, imitation of the manner should increase for both types of action. Not all kinds of interruptions may, however, lead to the successful segmentation and subsequent imitation of the manner. We therefore investigated whether a non-communicative signal - the actor paused, looked down and said 'Hmmm' - would lead to a similar increase in segmentation and manner imitation in a follow-up study. This way, we can infer whether children use such interruptions to segment and learn about actions and whether communicative signals are more suitable than other, non-communicative interruptions.

\section{Methods}

The methodology, hypotheses and analyses were preregistered on aspredicted.org, reference number \#5771 for the baseline and marked-communicative conditions and \#19880 for the marked-control condition. To account for missing data on the subject level, we deviated from the original hypothesis \#5771 by using a Generalised Linear Models with item and subject random effects instead of ANOVAs.

\section{Participants}

The final sample contained 60 18-month-old toddlers (Mean: 18m, Min: 17.5m, Max: 18.5m, 28 female), with 20 children in each condition. An additional 11 toddlers were tested, but excluded due to being unwilling to engage with the game (8), parental or sibling interference during all trials (2), incorrect age at time of testing (1). Eight of these children were in the communicative condition, 2 in the baseline condition and 1 in the non-communicative control condition. Written informed consent was provided by the caregivers and procedures were in accordance with institutional protocols.

\section{Materials}

The actions were presented on a green cardboard mat $(42 \times 60 \mathrm{~cm})$ with a small cardboard house (yellow, red). Four small toy animals (fox, rabbit, hedgehog, squirrel, all approximately $6-8 \mathrm{~cm}$ tall) were used to act out the actions. The animals were kept in a small, colourful box prior to the experiment. Additionally, we used a wooden stacking game during the warm-up phase.

\section{Procedure}

Toddlers were sitting on their caregiver's lap. After a warm-up session to familiarise the toddlers with the room and the experimenter, the experimenter presented each animal to the toddler with a short statement (e.g. "The squirrel has a bushy tail"). The toddler was allowed to play with all animals for approximately one minute. Afterwards, the animals were returned to the box and the experimenter revealed the board with the house. The modelling phase began during which the experimenter took out one animal, placed it on the 
board and said: "Look what the [animal] does!" (German original: "Schau mal, was das [Tier] macht!"). He then moved the animal across the table with either the sliding or the hopping action.

In the marked-communicatively condition, the experimenter looked up to the toddler and said "Wow" after the hopping/sliding movement, but before putting the animal into the house. In the baseline condition, the adult put the animal into the house before looking towards and addressing the child.

In the marked-control condition, the experimenter looked down, put his hand to the chin and said "Hmmmm." after the hopping/sliding movement, but before putting the animal into the house.

In the baseline condition, the experimenter did not pause between the hopping/sliding and putting animal into the house, but said "Wow" after putting the animal into the house to ensure the type and amount of verbal information remained the same when contrasted with the other conditions.

After the animal was put into the house, the experimenter said "Great, the animal went into the house. Now it's your turn!" (German original: "Toll, das Tier ist ins Haus gegangen. Jetzt bist du dran!") before pushing the board to the child. Each trial demonstration lasted approximately 10 seconds, and the child had 30 seconds to respond. If the child did not engage with the animal, the experimenter encouraged the child by saying "Now you can play with it!" ("Jetzt kannst du damit spielen"), "Now it's your turn" ("Jetzt bist du dran") or similar. If the child attempted to pull the house off the board, the experimenter said: "That's fixed." ("Das ist fest."). A visual illustration of the procedure is shown in Figure 1.

Each child was presented with up to four trials of the actions. The actions were shown in a fixed order of sliding-hopping-hopping-sliding, (as recommended by Southgate et al., 2009). Boundary-marking was presented as a between-subjects factor, and one third of the children saw the action in the boundary-marked communicatively condition, one third in the boundary-marked communicatively condition and one third in the boundary unmarked control condition.

\section{Coding}

Infants were scored on whether they (1) imitated the action manner (2) imitated the goal/outcome of the action. In line with previous research (Carpenter et al., 2005; Southgate et al., 2009), the action manner was coded as sliding when the animal moved continuously without breaking contact with the mat. The child imitated the hopping action, when the animal broke contact and made contact at least once again with the mat. The goal of putting the animal into the house was achieved if the child put the animal into the house at least once, even if the child removed the animal afterwards.

Children were included in the analysis if they contributed at least 2 out of the 4 trials. After coding, a total of 220 trials were included in the analysis. An additional 20 trials (9 hopping, 11 sliding) were excluded from the analysis due to the child refusing to touch the animal or being fussy (11), parental interference (7) and experimenter error (2). 


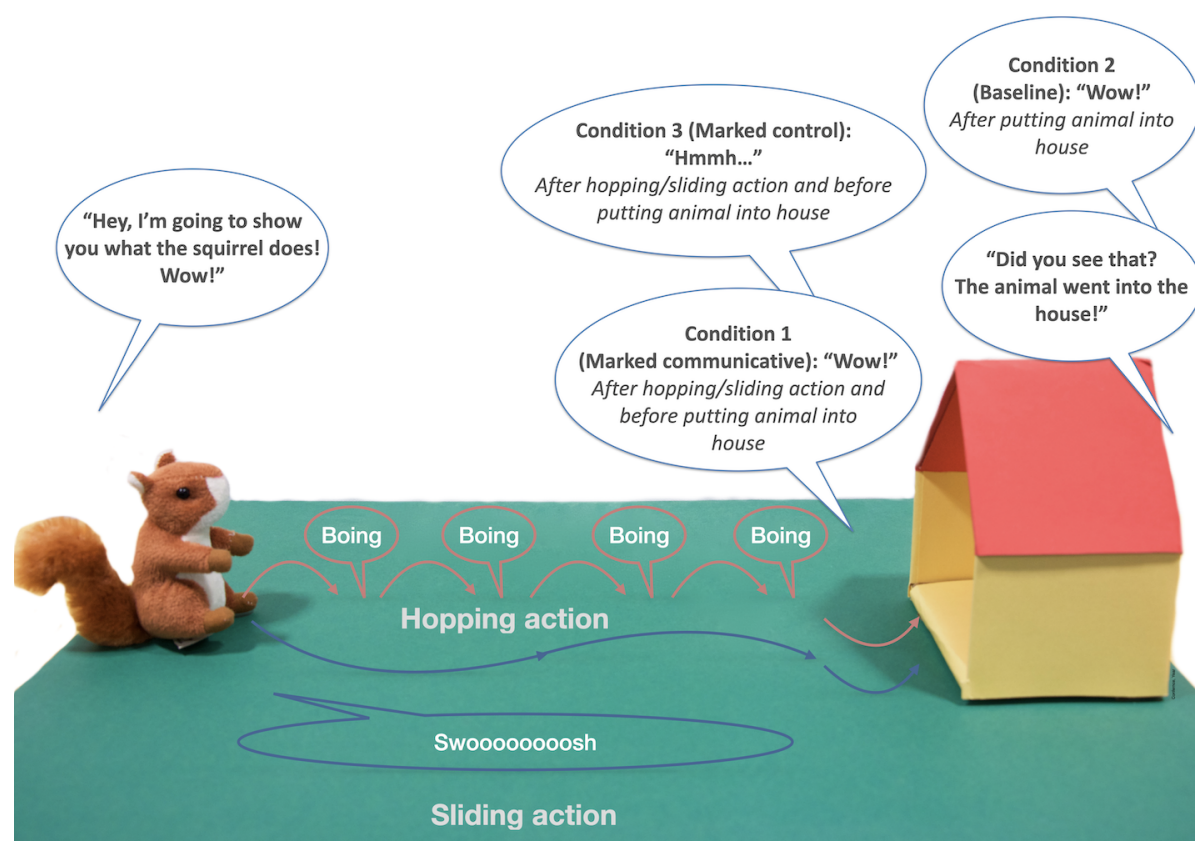

Figure 1. Illustration of the procedure and the three experimental conditions investigated. In the Baseline condition children were presented with the animal hopping or sliding into a house. In the Marked-Communicative condition, the action sequence was interrupted after the hopping and sliding but before putting the animal into the house, by the experimenter looking at the child and saying 'Wow!', i.e. signals commonly understood as ostensive. In the Marked-Control condition, the experimenter interrupted the action by saying 'Hmmmm!'. Crucially, the baseline condition also included an additional 'Wow!' at the end of the sequence, to control for the amount of ostensive signals children received.

A second coder naïve to the hypothesis coded manner and outcome in the videos of 39 children. The interrater agreement was excellent for both manner $\kappa=0.90, p<.0001$ and outcome $\kappa=0.94, p<0.001$.

\section{Data analysis}

Because some trials were missing, we decided to compute a Generalised Linear Mixed Effects model based on the binomial distribution using $\mathrm{R}$ (Version 4.0.3; R Core Team, 2019). Preprocessing was conducted using the tidyverse (Wickham et al., 2019) packages and statistical models were built in lme4 (Bates, Mächler, Bolker, \& Walker, 2015). Based on our pre-registration, we compared a model with Boundary Marker (factor levels: baseline, marked-communicative, marked-control) to a null model containing the intercept only. In an exploratory analysis, we also tested the interaction between Action Type (hopping/sliding) and Boundary Marking. We attempted to use the maximally converging random effects structure in line with recommendations by Barr, Levy, Scheepers, and Tily (2013). However, all models that were more complex than a single intercept with participant-ID at random effect level failed to converge due to singularity or the optimizer failing to establish a reliable solution to the model. The brms (Bürkner, 2017, 2018) and loo (Vehtari et al., 2020) 
Table 1

Model comparison between baseline, marked-communicative and marked-control condition for the first part of the action sequence, the manner of putting the animal into the house.

\begin{tabular}{lcccccccl}
\hline & npar & AIC & BIC & logLik & deviance & Chisq & Df & $\operatorname{Pr}(>$ Chisq $)$ \\
\hline mod.0 & 2.000 & 281.577 & 288.364 & -138.788 & 277.577 & NA & NA & NA \\
mod.marked & 4.000 & 282.082 & 295.656 & -137.041 & 274.082 & 3.495 & 2.000 & 0.174 \\
mod.interaction & 7.000 & 274.385 & 298.140 & -130.192 & 260.385 & 13.697 & 3.000 & 0.003 \\
\hline
\end{tabular}

packages were used to describe the model predictions using a bernoulli distribution and calculate Bayes Factors to compare the model's hypotheses.

\section{Results}

Manner imitation: We are primarily interested in whether children were more likely to imitate the first action step if the boundary between the two action steps was marked either communicatively or non-communicatively. The model containing only the boundary marker conditions did not perform better than the null model containing the interceptonly null model $\left(\chi^{2}(3.50)=2.00, p=.174\right)$, although the Bayesian analysis finds weak evidence in its favour $(B F=5)$. However, the model containing the interaction between boundary marker and action type performed significantly better than the marker-only model $\left(\chi^{2}(13.70)=3.00, p=.003, B F=3600\right)$ and the intercept only model $\left(\chi^{2}(17.19)=5.00\right.$, $p=.004, B F=19275)$. A detailed overview of the results can be seen in Table 1.

To investigate the effect of the boundary marking on children's imitation of the action manner, we first compared the manner imitation in the experimental groups to the baseline condition. To disentangle the interaction effect, we investigated these differences on subsets of the hopping/sliding action factor separately. Our results show that the sliding action was imitated by only few children at baseline $(\mathrm{M}=12 \%, 95 \% \mathrm{CI}[5 \%, 27 \%])$. Here, the marked-communicative condition shows a statistically significant increase in imitation (M $=39 \%, 95 \% \mathrm{CI}[24 \%, 58 \%], \beta=1.57, \mathrm{p}=.015)$, but this effect could not be found in the marked-control condition ( $\mathrm{M}=21 \%, 95 \% \mathrm{CI}[10 \%, 39 \%], \beta=0.72, \mathrm{p}=.271)$. The hopping action was imitated by half the children at baseline level $(\mathrm{M}=50 \%, 95 \% \mathrm{CI}[33 \%, 67 \%])$. Marking the boundary communicatively $(\mathrm{M}=42 \%, 95 \% \mathrm{CI}[26 \%, 59 \%])$ or with the control intervention $(\mathrm{M}=29 \%, 95 \% \mathrm{CI}[16 \%, 46 \%])$ did not significantly affect their imitation (Baseline - Marked-communicative: $\beta=-0.38, \mathrm{p}=.524$, Baseline - Marked-control: $\beta=$ $-1.05, \mathrm{p}=.103)$. A visual representation of these results can be found in Figure 2.

We investigated the effect of communication against the other two conditions by investigating subsets of the hopping and sliding data. We find that children imitate the sliding manner significantly more $(\beta=1.20, \mathrm{p}=.022)$ than in the other two conditions, but marking actions in the communicative condition does not have the same effect for the hopping action $(\beta=0.13, \mathrm{p}=.797)$.

Finally, we analysed the difference between the hopping/sliding action for each of the condition levels separately. In line with previous studies using this paradigm (Carpenter et 


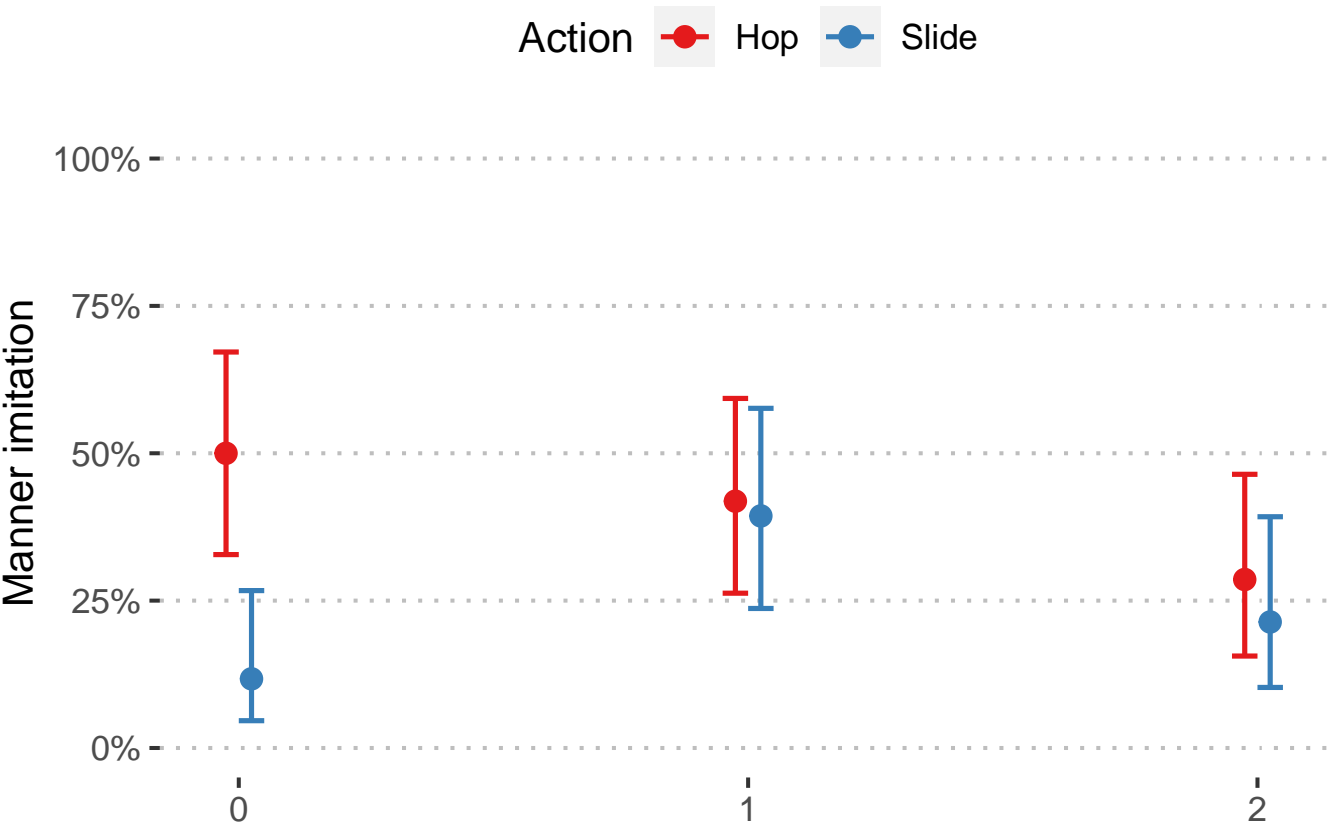

Figure 2. Predicted manner of imitation in the first action unit, the action manner (hopping/sliding) for the baseline, marked-communicative and marked-control conditions. Error bars indicate upper and lower confidence intervals.

al., 2005) we found a difference in the imitation of the hopping and sliding action in the baseline condition, i.e. the condition that replicates the original study most closely $(\beta=$ $-2.01, \mathrm{p}=.002)$. This difference was neither apparent in the marked-communicative $(\beta=$ $-0.10, \mathrm{p}=.843)$, nor the marked-control condition $(\beta=-0.41, \mathrm{p}=.473)$.

Outcome imitation Imitation of the action outcome was very high across all conditions and $0.91 \%$ of the children imitated the outcome. Adding additional factors to account for action type, boundary marking and their corresponding interaction did not lead to a significantly better model.

\section{Discussion}

We were interested in the role of communicative and non-communicative signals in segmenting action sequences during child-directed action presentations. Our results showed that separating a short action sequence by briefly interrupting the action steps using childdirected speech and direct gaze increased children's imitation of the less salient sliding action. For the more salient hopping action, marking the boundary between the action manner and the outcome did not increase the level of imitation that was already high in the unmarked condition. However, we do not find such an effect for the non-communicatively marked control condition. These results provide support for the hypothesis that communicative signals help children segment action sequences, but these effects are only observable in less salient actions.

Our results suggest that any effect of ostensive signals as event boundary markers 
only generalises to the sliding action in our study. In contrast, the hopping action remained unaffected, and was copied at a comparatively high level independent of condition. It is possible that the hopping action used in our study is more readily identified as an action unit, due to its salience. Previous research has already highlighted the role of salience in children's imitation of actions. For example, toddlers between 12-30 months were more likely to imitate a hammering action compared to a less salient pulling action (Gampe, Prinz, \& Daum, 2016) and 12-month-olds were better at learning to anticipate reaches towards large, compared to small objects (Adam et al., 2016; Henrichs, Elsner, Elsner, \& Gredebäck, 2012). Therefore, the contribution of communicative signals to goal-directed action segmentation might be to identify action boundaries, particularly in low salience actions.

These results are compatible with theories emphasising the segmentation and chunking of incoming information, such as Event Segmentation Theory (Zacks et al., 2007; Zacks \& Swallow, 2007) and domain-general theories of chunking and bottlenecks (Christiansen \& Chater, 2016; Isbilen, McCauley, Kidd, \& Christiansen, 2020). Event Segmentation Theory also suggests that bottom-up and top-down processes influence the perception of an incoming stream of events (Zacks \& Swallow, 2007), such as the action sequence observed by the children in our study. According to Event Segmentation Theory, the way that an event is segmented affects how it is interpreted. Consequently, the segmentation of an action should lead to a different interpretation of the action sequence. Whereas the uninterrupted action sequence is interpreted as "putting the animal into the house" (by any means), the interrupted event sequence is interpreted as two separate events of "sliding" and "putting the animal into the house". Crucially, because the hopping action is more readily recognised, more salient, more repetitive and potentially has clearer event boundaries, the hopping action may have already provided sufficient information to be recognised as its own separate action sequence, and additional segmentation cues were not helpful.

What then caused the increased imitation of the sliding action preceding the communicative signals, but not a non-communicative control cue? These differences can be explained by appealing to low-level features of direct gaze and infant-directed speech, as well as higher order inferences about the communicative intent of the interruption.

Social signals share certain properties that make them particularly useful for this purpose. For example, infants show a stimuli-specific preference towards gaze (Farroni, Csibra, Simion, \& Johnson, 2002; Farroni, Johnson, Brockbank, \& Simion, 2000; Michel et al., 2017a, 2017b) and infant-directed speech (Dominey \& Dodane, 2004) and direct gaze appears to interrupt working memory in adults (Wang \& Apperly, 2016). However, so far we do not know why these specific properties of direct gaze and child-directed-speech make them suitable for action and event segmentation. In fact, other, non-social signals may have similar effects on action segmentation and increase the imitation of action manner in this paradigm as long as the boundary-marking event is clearly identifiable as an intentional action. For instance, it is possible that a non-social, but clearly intentional 'beep' initiated by a button press may have had a similar effect on action segmentation, comparable to the way that children flexibly use an adult's intentional placement of an item as a referential signal to identify the location of a hidden reward (Moore, Liebal, \& Tomasello, 2013; Moore, Mueller, Kaminski, \& Tomasello, 2015). If this is the case, even a simple pause might be 
sufficient to induce the segmentation of an action. For example, 6- (Sharon \& Wynn, 1998) and 10- to 11-month old (Baldwin et al., 2001) infants that have been familiarised with video sequences of everyday actions look longer if the video sequence is paused within an intentional action, compared to a pause between intentional actions. Therefore, the effect might not be specific to social signals and any pause may be sufficient to segment non-salient actions for children and subsequently increase their imitation.

Why was imitation not affected in the non-communicative control condition in this study? It is likely that communicative signals are particularly suitable in segmenting action sequences, either because of their privileged role as communicative markers in general (Farroni et al., 2005; Senju, Johnson, \& Csibra, 2006), or because they interrupt working memory (Wang \& Apperly, 2016). However, we cannot rule out the possibility that other non-communicative markers are also suitable for segmenting action sequences. It is possible that children interpreted the non-communicative control marker to indicate hesitation, marking the preceding action as less intentional or even accidental. In other studies, children of a similar age were sensitive to such information and imitated the intended goals of failed actions, instead of faithfully copying accidental actions (Carpenter, Akhtar, \& Tomasello, 1998). Therefore, they may have interpreted the part of the action preceding the "Hmmm" as accidental and non-intentional, and were therefore less inclined to imitate it.

The communicative interruption of the action sequence in our study does not support the interpretation of direct gaze and social signals as markers of ostension in this particular context. According to Natural Pedagogy, communicative signals function like a 'switch' (Csibra, 2010) for learning that is either on or off instead of providing a gradual response towards different levels of "communicative-ness" (Parise \& Csibra, 2013). There are three reasons, why such an interpretation is unlikely (1) In our study, the entire action sequence was sandwiched between the experimenter speaking to the child using child-directed and ostensive communication at the beginning and the end of each trial. (2) The amount of communicative signals remained the same, and only the location of one of them was varied. (3) Communicative signals typically precede relevant information, but in our study they followed the part of the action sequence that children were more likely to imitate. Therefore, the structure of the task did not make the communicative marker a signal of ostension. Instead, children may have used the already established relevance of the action demonstration to interpret the position of the communicative marker.

Whether we appeal to a lower-level or a higher-level interpretation of the resultsinterrupting the action sequence communicatively provides important information on interpreting the meaning of the events by providing temporal reference, in addition to spatial reference that can be established through referential gaze to object locations (Butler, Caron, \& Brooks, 2000) or pointing (Gliga \& Csibra, 2009; Melinder, Konijnenberg, Hermansen, Daum, \& Gredebäck, 2014; Morissette et al., 1995). In just the same way that gaze and pointing can be used to indicate the location of an object in space, ostensive signals can be used to signal event boundaries. Following lower-level accounts, such as cognitive chunking, the communicative interruption provided a possibility to store the manner of the action separately rather than chunk it together with the more salient goal and subsequently forget it. According to the higher-level inferential account provided by Natural Pedagogy, children 
actively searched for the relevance of the communicative interruption.

Finally, as in previous studies (Carpenter et al., 2005; Southgate et al., 2009), children imitated the outcome to a very high degree and our manipulation did not affect their imitation of the outcome of the action sequence, i.e. putting the animal into the house. Previous studies attributed this difference to the importance of distinct goals versus the manner of the action. This description is compatible with an event segmentation description. In addition, interpreting these findings using event segmentation theory allows us to provide a general mechanism of explaining why children chose to imitate one part of the action, but not the other. Here, children do not segment both parts of the action sequence (comprising of the manner and outcome) and only imitate the most salient part of the action, the outcome.

\section{Conclusions}

Our study shows that communicative signals can help to increase the imitation of a nonsalient action unit in 18-month-old children. Importantly, our results also show that children do not use any interruption to segment these actions. In our study, the communicative marker, but not the non-communicative control marker increased the imitation of the sliding action. These findings open up a new way of looking at the role of caregiver-child interactions by highlighting the role of structural information that guides pedagogical actions.

The preceding discussion of our results has also shown the importance of further investigating how children use social and non-social signals to segment events. As the results of our experiment only show an effect of marker location on one of the two actions, future investigations need to systematically broaden the range of actions and control their salience to generalise the findings to other actions.

In the current study we investigated the effect of communicative signals on the segmentation of a short action sequence. We have argued that this may be a crucial aspect of teaching novel actions to children. However, it is likely that these signals may also be useful in segmenting events in general, for example when teaching verbs.

Additionally, by looking at the information provided by caregivers in action teaching contexts, we find many similarities with the segmentation and processing of linguistic information (see Christiansen \& Chater, 2016). Processing of action steps may be incremental and lower levels of representation may be chunked together to form larger representations of actions and their meanings. Children as well as adults may use such an incremental, bottom-up approach to identify and segment basic action units and re-assemble them into larger chunks to facilitate prediction. By drawing on the structural information that is inherent in action-, event- and language processing, it may be possible to develop and enhance domain-general models of learning.

\section{References}

Adam, M., Reitenbach, I., Papenmeier, F., Gredebäck, G., Elsner, C., \& Elsner, B. (2016). Goal saliency boosts infants' action prediction for human manual actions, but not for mechanical claws. Infant Behavior and Development, 44, 29-37. https://doi.org/10.101 6/j.infbeh.2016.05.001 
Baldwin, D. A., Baird, J. A., Saylor, M. M., \& Clark, M. A. (2001). Infants parse dynamic action. Child Development, 72(3), 708-717. https://doi.org/10.1111/1467-8624.00310

Barr, D. J., Levy, R., Scheepers, C., \& Tily, H. J. (2013). Random effects structure in mixedeffects models: Keep it maximal. Journal of Memory and Language, 68(3), 255-278. https://doi.org/10.1016/j.jml.2012.11.001

Bates, D., Mächler, M., Bolker, B., \& Walker, S. (2015). Fitting linear mixed-effects models using lme4. Journal of Statistical Software, 67(1), 1-48. https://doi.org/10.18637/jss.v 067.i01

Bavelas, J. B., Coates, L., \& Johnson, T. (2002). Listener responses as a collaborative process: The role of gaze. Journal of Communication, 52(3), 566-580. https://doi.org/ 10.1111/j.1460-2466.2002.tb02562.x

Biro, S. (2013). The role of the efficiency of novel actions in infants' goal anticipation. Journal of Experimental Child Psychology, 116(2), 415-427. https://doi.org/10.1016/j.je cp.2012.09.011

Biro, S., Verschoor, S., Coalter, E., \& Leslie, A. M. (2014). Outcome producing potential influences twelve-month-olds' interpretation of a novel action as goal-directed. Infant Behavior and Development, 37(4), 729-738. https://doi.org/10.1016/j.infbeh.2014.09.004

Brand, R. J., Baldwin, D. A., \& Ashburn, L. A. (2002). Evidence for "motionese": Modifications in mothers' infant-directed action. Developmental Science, 5(1), 72-83. https://doi.org/10.1016/j.infbeh.2003.09.004

Brand, R. J., Hollenbeck, E., \& Kominsky, J. F. (2013). Mothers' infant-directed gaze during object demonstration highlights action boundaries and goals. IEEE Transactions on Autonomous Mental Development, 5(3), 192-201. https://doi.org/10.1109/TAMD.2013. 2273057

Brand, R. J., McGee, A., Kominsky, J. F., Briggs, K., Gruneisen, A., \& Orbach, T. (2009). Repetition in infant-directed action depends on the goal structure of the object: Evidence for statistical regularities. Gesture, 9(3), 337-353. https://doi.org/10.1075/gest.9.3.04bra

Brand, R. J., Shallcross, W. L., Sabatos, M. G., \& Massie, K. P. (2007). Fine-grained analysis of motionese: Eye gaze, object exchanges, and action units in infant-versus adult-directed action. Infancy, 11 (2), 203-214. https://doi.org/10.1111/j.1532-7078.2007.tb00223.x

Butler, S. C., Caron, A. J., \& Brooks, R. (2000). Infant understanding of the referential nature of looking. Journal of Cognition and Development, 1(4), 359-377. https: //doi.org/10.1207/S15327647JCD0104_01

Bürkner, P.-C. (2017). brms: An R package for Bayesian multilevel models using Stan. Journal of Statistical Software, 80(1), 1-28. https://doi.org/10.18637/jss.v080.i01

Bürkner, P.-C. (2018). Advanced Bayesian multilevel modeling with the R package brms. The R Journal, 10(1), 395-411. https://doi.org/10.32614/RJ-2018-017

Carpenter, M., Akhtar, N., \& Tomasello, M. (1998). Fourteen- through 18-month-old 
infants differentially imitate intentional and accidental actions. Infant Behavior and Development, 21 (2), 315-330. https://doi.org/10.1016/s0163-6383(98)90009-1

Carpenter, M., Call, J., \& Tomasello, M. (2005). Twelve- and 18-month-olds copy actions in terms of goals. Developmental Science, 8(1), F13-F20. https://doi.org/10.1111/j.14677687.2004.00385.x

Christiansen, M. H., \& Chater, N. (2016). The now-or-never bottleneck: A fundamental constraint on language. Behavioral and Brain Sciences, 39, 1-72. https://doi.org/10.101 $7 / \mathrm{s} 0140525 \mathrm{x} 1500031 \mathrm{x}$

Clark, H. H. (2003). Pointing and placing. In S. Kita (Ed.), Pointing. Where language, culture, and cognition meet (pp. 243-268). Hillsdale NJ: Erlbaum.

Csibra, G. (2003). Teleological and referential understanding of action in infancy. Philosophical Transactions of the Royal Society of London. Series B: Biological Sciences, 358(1431), 447-458. https://doi.org/10.1098/rstb.2002.1235

Csibra, G. (2008). Action mirroring and action understanding: An alternative account. In P. Haaggard, Y. Rossetti, \& M. Kawato (Eds.), Attention and performance XXII: Sensorymotor foundations of higher cognition (pp. 435-459). Oxford (UK): Oxford University Press.

Csibra, G. (2010). Recognizing communicative intentions in infancy. Mind \& Brain, 25(2), 141-168. https://doi.org/10.1111/j.1468-0017.2009.01384.x

Csibra, G., \& Gergely, G. (2009). Natural pedagogy. Trends in Cognitive Sciences, 13, 148-153. https://doi.org/10.1016/j.tics.2009.01.005

Csibra, G., \& Gergely, G. (2011). Natural pedagogy as evolutionary adaptation. Philosophical Transactions of the Royal Society B: Biological Sciences, 366(1567), 1149-1157. https: //doi.org/10.1098/rstb.2010.0319

Csibra, G., \& Shamsudheen, R. (2015). Nonverbal generics: Human infants interpret objects as symbols of object kinds. Annual Review of Psychology, 66(1), 689-710. https://doi.org/10.1146/annurev-psych-010814-015232

Dominey, P. F., \& Dodane, C. (2004). Indeterminacy in language acquisition: The role of child directed speech and joint attention. Journal of Neurolinguistics, 17(2-3), 121-145. https://doi.org/10.1016/S0911-6044(03)00056-3

Elman, J. L. (1990). Finding structure in time. Cognitive Science, 14(2), 179-211. https: //doi.org/10.1207/s15516709cog1402_1

Farroni, T., Csibra, G., Simion, F., \& Johnson, M. H. (2002). Eye contact detection in humans from birth. Proceedings of the National Academy of Sciences, 99(14), 9602-9605. https://doi.org/10.1073/pnas.152159999

Farroni, T., Johnson, M. H., Brockbank, M., \& Simion, F. (2000). Infants' use of gaze direction to cue attention: The importance of perceived motion. Visual Cognition, 7(6), 705-718. https://doi.org/10.1080/13506280050144399 
Farroni, T., Johnson, M. H., Menon, E., Zulian, L., Faraguna, D., \& Csibra, G. (2005). Newborns' preference for face-relevant stimuli: Effects of contrast polarity. Proceedings of the National Academy of Sciences, 102(47), 17245-17250. https://doi.org/10.1073/pn as.0502205102

Fausey, C. M., Jayaraman, S., \& Smith, L. B. (2016). From faces to hands: Changing visual input in the first two years. Cognition, 152, 101-107. https://doi.org/10.1016/j.cognitio n.2016.03.005

Gampe, A., Prinz, W., \& Daum, M. M. (2016). Measuring action understanding: Relations between goal prediction and imitation. British Journal of Developmental Psychology, 34 (1), 53-65. https://doi.org/10.1111/bjdp.12125

Gergely, G., Bekkering, H., \& Kiraly, I. (2002). Rational imitation in preverbal infants. Nature, 415 (6873), 755-755. https://doi.org/10.1038/415755a

Gergely, G., \& Csibra, G. (2003). Teleological reasoning in infancy: The naïve theory of rational action. Trends in Cognitive Sciences, 7(7), 287-292. https://doi.org/10.1016/S1 364-6613(03)00128-1

Gliga, T., \& Csibra, G. (2009). One-year-old infants appreciate the referential nature of deictic gestures and words. Psychological Science, 20(3), 347-353. https://doi.org/10.1 111/j.1467-9280.2009.02295.x

Goldstein, M. H., Waterfall, H. R., Lotem, A., Halpern, J. Y., Schwade, J. A., Onnis, L., \& Edelman, S. (2010). General cognitive principles for learning structure in time and space. Trends in Cognitive Sciences, 14(6), 249-258. https://doi.org/10.1016/j.tics.2010.02.004

Gredebäck, G., \& Melinder, A. (2011). Teleological reasoning in 4-month-old infants: Pupil dilations and contextual constraints. PLoS ONE, 6(10), e26487. https://doi.org/10.137 1/journal.pone.0026487

Gredebäck, G., Stasiewicz, D., Falck-Ytter, T., Rosander, K., \& Hofsten, C. von. (2009). Action type and goal type modulate goal-directed gaze shifts in 14-month-old infants. Developmental Psychology, 45(4), 1190-1194.

Henrichs, I., Elsner, C., Elsner, B., \& Gredebäck, G. (2012). Goal salience affects infants' goaldirected gaze shifts. Frontiers in Psychology, 3. https://doi.org/10.3389/fpsyg.2012.00391

Hespos, S. J., Grossman, S. R., \& Saylor, M. M. (2010). Infants' ability to parse continuous actions: Further evidence. Neural Networks, 23(8-9), 1026-1032. https://doi.org/10.101 6/j.neunet.2010.07.010

Hespos, S. J., Saylor, M. M., \& Grossman, S. R. (2009). Infants' ability to parse continuous actions. Developmental Psychology, 45(2), 575-585. https://doi.org/10.1037/a0014145

Hommel, B., Müsseler, J., Aschersleben, G., \& Prinz, W. (2001). The theory of event coding (TEC): A framework for perception and action planning. Behavioral and Brain Sciences, 24(5), 849-878. https://doi.org/10.1017/s0140525x01000103

Hömke, P., Holler, J., \& Levinson, S. C. (2017). Eye blinking as addressee feedback in 
face-to-face conversation. Research on Language and Social Interaction, 50(1), 54-70. https://doi.org/10.1080/08351813.2017.1262143

Isbilen, E. S., McCauley, S. M., Kidd, E., \& Christiansen, M. H. (2020). Statistically induced chunking recall: A memory-Based approach to statistical learning. Cognitive Science, 44 (7). https://doi.org/10.1111/cogs.12848

Jones, S. S. (2007). Imitation in infancy: The development of mimicry. Psychological Science, 18(7), 593-599. https://doi.org/10.1111/j.1467-9280.2007.01945.x

Jones, S. S. (2009). The development of imitation in infancy. Philosophical Transactions of the Royal Society B: Biological Sciences, 364(1528), 2325-2335. https://doi.org/10.1098/ rstb.2009.0045

Király, I., Csibra, G., \& Gergely, G. (2013). Beyond rational imitation: Learning arbitrary means actions from communicative demonstrations. Journal of Experimental Child Psychology, 116(2), 471-486. https://doi.org/10.1016/j.jecp.2012.12.003

Koterba, E. A., \& Iverson, J. M. (2009). Investigating motionese: The effect of infant-directed action on infants' attention and object exploration. Infant Behavior and Development, 32(4), 437-444. https://doi.org/10.1016/j.infbeh.2009.07.003

Melinder, A. M. D., Konijnenberg, C., Hermansen, T., Daum, M. M., \& Gredebäck, G. (2014). The developmental trajectory of pointing perception in the first year of life. Experimental Brain Research, 233(2), 641-647. https://doi.org/10.1007/s00221-014-4143-2

Michel, C., Pauen, S., \& Hoehl, S. (2017a). Schematic eye-gaze cues influence infants' object encoding dependent on their contrast polarity. Scientific Reports, 7(1). https: //doi.org/10.1038/s41598-017-07445-9

Michel, C., Wronski, C., Pauen, S., Daum, M. M., \& Hoehl, S. (2017b). Infants' object processing is guided specifically by social cues. Neuropsychologia, 126, 54-61. https: //doi.org/10.1016/j.neuropsychologia.2017.05.022

Moore, R., Liebal, K., \& Tomasello, M. (2013). Three-year-olds understand communicative intentions without language, gestures, or gaze. Interaction Studies, 14(1), 62-80. https: //doi.org/10.1075/is.14.1.05moo

Moore, R., Mueller, B., Kaminski, J., \& Tomasello, M. (2015). Two-year-old children but not domestic dogs understand communicative intentions without language, gestures, or gaze. Developmental Science, 18(2), 232-242. https://doi.org/10.1111/desc.12206

Morissette, P., Ricard, M., \& Décarie, T. G. (1995). Joint visual attention and pointing in infancy: A longitudinal study of comprehension. British Journal of Developmental Psychology, 13(2), 163-175. https://doi.org/10.1111/j.2044-835X.1995.tb00671.x

Parise, E., \& Csibra, G. (2013). Neural responses to multimodal ostensive signals in 5-monthold infants. PLoS ONE, 8(8), e72360. https://doi.org/10.1371/journal.pone.0072360

R Core Team. (2019). R: A language and environment for statistical computing. Vienna, Austria: R Foundation for Statistical Computing. Retrieved from https://www.R- 
project.org/

Reid, V. M., Hoehl, S., Grigutsch, M., Groendahl, A., Parise, E., \& Striano, T. (2009). The neural correlates of infant and adult goal prediction: Evidence for semantic processing systems. Developmental Psychology, 45(3), 620-629. https://doi.org/10.1037/a0015209

Rutherford, M. D., \& Przednowek, M. (2012). Fathers show modifications of infant-directed action similar to that of mothers. Journal of Experimental Child Psychology, 111 (3), 367-378. https://doi.org/10.1016/j.jecp.2011.10.012

Sage, K., \& Baldwin, D. (2012). Exploring natural pedagogy in play with preschoolers: Cues parents use and relations among them. Education Research and Perspectives, 39(1), $153-181$.

Schaik, J. E. van, Meyer, M., Ham, C. R. van, \& Hunnius, S. (2020). Motion tracking of parents' infant- versus adult-directed actions reveals general and action-specific modulations. Developmental Science, 23(1), e12869. https://doi.org/10.1111/desc.12869

Senju, A., \& Csibra, G. (2008). Gaze following in human infants depends on communicative signals. Current Biology, 18, 668-671. https://doi.org/10.1016/j.cub.2008.03.059

Senju, A., Csibra, G., \& Johnson, M. H. (2008). Understanding the referential nature of looking: Infants' preference for object-directed gaze. Cognition, 108, 303-319. https: //doi.org/10.1016/j.cognition.2008.02.009

Senju, A., Johnson, M. H., \& Csibra, G. (2006). The development and neural basis of referential gaze perception. Social Neuroscience, 1(3-4), 220-234. https://doi.org/10.108 0/17470910600989797

Sharon, T., \& Wynn, K. (1998). Individuation of actions from continuous motion. Psychological Science, 9(5), 357-362. https://doi.org/10.1111/1467-9280.00068

Southgate, V., Chevallier, C., \& Csibra, G. (2009). Sensitivity to communicative relevance tells young children what to imitate. Developmental Science, 12, 1013-1019. https: //doi.org/10.1111/j.1467-7687.2009.00861.x

Sperber, D., \& Wilson, D. (1995). Relevance: Communication and cognition (2nd ed.). Maiden, MA: Wiley-Blackwell.

Sperber, D., \& Wilson, D. (2002). Pragmatics, modularity and mind-reading. MindE Language, 17(1-2), 3-23. https://doi.org/10.1111/1468-0017.00186

Twomey, K. E., Ma, L., \& Westermann, G. (2017). All the right noises: Background variability helps early word learning. Cognitive Science. https://doi.org/10.1111/cogs.1 2539

Twomey, K. E., \& Westermann, G. (2017). Curiosity-based learning in infants: A neurocomputational approach. Developmental Science, 21(4), e12629. https://doi.org/10.1111/de sc. 12629

Vehtari, A., Gabry, J., Magnusson, M., Yao, Y., Bürkner, P.-C., Paananen, T., \& Gelman, A. (2020). Loo: Efficient leave-one-out cross-validation and WAIC for bayesian models. 
Retrieved from https://mc-stan.org/loo

Verschoor, S. A., Spapé, M., Biro, S., \& Hommel, B. (2013). From outcome prediction to action selection: Developmental change in the role of action-effect bindings. Developmental Science, 16(6), 801-814. https://doi.org/10.1111/desc.12085

Wang, J. J., \& Apperly, I. A. (2016). Just one look: Direct gaze briefly disrupts visual working memory. Psychonomic Bulletin\& Review, 24(2), 393-399. https://doi.org/10.3 758/s13423-016-1097-3

Wickham, H., Averick, M., Bryan, J., Chang, W., McGowan, L. D., François, R., ... Yutani, H. (2019). Welcome to the tidyverse. Journal of Open Source Software, 4(43), 1686. https://doi.org/10.21105/joss.01686

Williamson, R. A., \& Brand, R. J. (2014). Child-directed action promotes 2-year-olds' imitation. Journal of Experimental Child Psychology, 118, 119-126. https://doi.org/10.1 016/j.jecp.2013.08.005

Woodward, A. L., \& Guajardo, J. J. (2002). Infants' understanding of the point gesture as an object-directed action. Cognitive Development, 17(1), 1061-1084. https://doi.org/10 $.1016 / \mathrm{s} 0885-2014(02) 00074-6$

Zacks, J. M. (2004). Using movement and intentions to understand simple events. Cognitive Science, 28(6), 979-1008. https://doi.org/10.1016/j.cogsci.2004.06.003

Zacks, J. M., Kumar, S., Abrams, R. A., \& Mehta, R. (2009). Using movement and intentions to understand human activity. Cognition, 112(2), 201-216. https://doi.org/10.1016/j.co gnition.2009.03.007

Zacks, J. M., Speer, N. K., Swallow, K. M., Braver, T. S., \& Reynolds, J. R. (2007). Event perception: A mind-brain perspective. Psychological Bulletin, 133(2), 273-293. https://doi.org/10.1037/0033-2909.133.2.273

Zacks, J. M., \& Swallow, K. M. (2007). Event segmentation. Current Directions in Psychological Science, 16(2), 80-84. https://doi.org/10.1111/j.1467-8721.2007.00480.x

Zacks, J. M., \& Tversky, B. (2001). Event structure in perception and conception. Psychological Bulletin, 127(1), 3-21. https://doi.org/10.1037/0033-2909.127.1.3

Zacks, J. M., Tversky, B., \& Iyer, G. (2001). Perceiving, remembering, and communicating structure in events. Journal of Experimental Psychology: General, 130(1), 29-58. https://doi.org/10.1037/0096-3445.130.1.29 